\title{
Potential roles of STAT3 in control of differentiation of hematopoietic and neuronal progenitors
}

\author{
Xin-Yuan Fu ${ }^{1}$, Wen-Jun Zhang ${ }^{1}$, Scott Cooper ${ }^{1}$, Giao Hangoc ${ }^{1}$, Akira Moh ${ }^{1}$, Hal E Broxmeyer ${ }^{1}$ \\ ${ }^{1}$ Microbiology and Immunology, Walther Oncology Center, Indiana University School of Medicine, Indianapolis, IN, USA
}

It is known that STAT3 has an essential function in LIF-mediated maintaining of mouse embryonic stem cells. However, it is unclear what are the functional roles of STAT3 in adult stem cells/early progenitors. Because functional deletion of STAT3 in mice is embryonic lethal, it was difficult to evaluate the roles of STAT3 in adult. To address this problem, we have generated a number of mouse stains of tissue specific disruption of STAT3, including in bone marrow/hematopoietic lineages (Welte et al., PNAS 100: 1879, 2003) and in neuronal lineages (Gao et al., PNAS 101: 4661, 2004). The availability of these conditional STAT3 -/- mouse models have provided tools for studies of functional roles of STAT3 during cell differentiation from early progenitor cells. We have evaluated potential roles for STAT3 in hematopoiesis after birth, and found that STAT3 would be one critical factor involved in the proliferation of myeloid progenitor cells (MPC: CFU-GM, BFU-E, CFU-GEMM) in bone marrow and spleen. Particularly, we found that deletion of STAT3 after birth results in marked decreases in absolute numbers and cell cycling status of marrow and spleen myeloid progenitor cells, and STAT3 appeared to be a critical molecule in mediating synergistically stimulated progenitor cell proliferation. In the studies of neuronal lineage, we have found that highly tyrosine-phosphorylated STAT3 is often present and enriched in regions of neuronal precursor cells/layers (such as in subventricular areas) but is transiently disappeared in differentiated cells/layers. This observation suggested a possible role of STAT3 in regulation of neuronal precursors. To further study the role of STAT3, we generated neuronal STAT3 -/- mouse (using floxed STAT3 and Nestin-cre). In particular, the effect of STAT3 deletion in vivo on astrocyte differentiation was examined. We found that deletion of STAT3 alone has a limited effect on astrocyte differentiation as measure by GFAP expression. However, the double deletion of STAT3 and STAT1 has almost completely abolished GFAP expression, indicating STAT3 and STAT1 has a redundant role during astrocyte differentiation. The details of our findings will be further discussed in the meeting.

Keywords: STAT3, progenitors, hematopoietic, neuronal lineages

Cell Research (2008) 18:s14. doi: 10.1038/cr.2008.104; published online 4 August 2008

Correspondence: Xin-Yuan Fu

E-mail: xfu@iupui.edu

Xin-Yuan $\mathrm{Fu}, \mathrm{PhD}$, is currently Professor of Immunology at Indiana University School of Medicine USA. He received his $\mathrm{PhD}$ in molecular biology at Columbia University in 1988 (Mentor: James Manley); postdoctoral training in Rockefeller University with James Darnell (19881991). He was an Assistant Professor in Mt Sinai School of Medicine NY (1992-1994); an Associate Professor at Yale University (1994-2003), a Full Professor at Indiana University (2004-present). Dr Fu served as member of Hem 1/Hematopoiesis Study Section of NIH (1999-2004). One of Dr
Fu's contributions to science is the discovery STAT gene family ( $\mathrm{Fu}$ et al., 1992, Proc Natl Acad Sci USA, 89:7840-7843) while he was working in Darnell's laboratory, and the revelation of the molecular mechanism of signal transduction through the STAT pathway (Fu, 1992, Cell, 70:323335). In the past ten years his work has focused on STAT3's role in regulation of inflammation, dendritic cell differentiation and development. Currently, one of Dr Fu's research interests is to reveal roles of the STAT gene family in epigenetic regulation of stem cell differentiation and development. Please visit Dr Fu's laboratory website for more information on his research interests (http://www.iupui.edu/ micro/fu.html). 